\title{
On Rules and Parameter Free Systems in Bounded Arithmetic
}

\author{
Andres Cordòn-Franco, Alejandro Fernández-Margarit, \\ and Francisco Felix Lara-Martín* \\ Facultad de Matemáticas. Universidad de Sevilla \\ C/ Tarfia, s/n, 41012 Sevilla, Spain \\ \{acordon, afmargarit,fflara\}@us.es
}

\begin{abstract}
We present model-theoretic techniques to obtain conservation results for first order bounded arithmetic theories, based on a hierarchical version of the well known notion of an existentially closed model.
\end{abstract}

Keywords: Bounded Arithmetic, conservation results, parameter free schemes.

\section{Introduction}

Bounded arithmetic theories are formal systems tailored to capture computational complexity classes. The foundational work in this area is [3], where S. Buss introduced the families of theories $S_{2}^{i}$ and $T_{2}^{i}(i \geq 0)$ and showed that they can be considered as formal counterparts of the Polynomial Time Hierarchy $P H$. Since then a variety of related systems have been introduced in order to deal with other complexity classes. Among the fundamental results on these systems two groups can be isolated: (a) characterizations of their computational strength, mainly, by determining their $\Sigma_{i}^{b}$-definable functions; and (b) relationship among different axiomatizations, especially, conservation results.

Here we present model-theoretic methods to obtain both kinds of results for restricted versions of Buss's theories $S_{2}^{i}, T_{2}^{i}$ as well as for the $\Sigma_{i}^{b}$-replacement scheme $B B \Sigma_{i}^{b}$. Systems $S_{2}^{i}$ and $T_{2}^{i}$ are axiomatized over a certain base theory by axiom schemes expressing (respectively) the polynomial and the usual induction principles restricted to $\Sigma_{i}^{b}$-formulas. We shall weaken these theories in two ways: (1) by formalizing the corresponding induction or replacement principle as an inference rule instead of an axiom scheme, or (2) by restricting the induction schemes to parameter free formulas. In the first case we drop the axiom scheme and consider the closure of the base theory under first order logic and nested applications of the corresponding inference rule. In the second case we still deal with an axiom scheme but now it is restricted to formulas with no other free variables than the induction variable. While the effects of these restrictions have been extensively investigated for fragments of Peano Arithmetic, it is not the

^ Partially supported by grants MTM2005-08658 of MEC and TIC-137 of Junta de Andalucía, Spain. Part of this work was done while the first author was visiting the Mathematical Institute of the Academy of Sciences of the Czech Republic in 2004. 
case of Bounded Arithmetic. To our best knowledge, parameter free systems have only been systematically studied by S. Bloch in the second part of his thesis 2. On the other hand, systems described by inference rules in the sense above seldom appear in an explicit manner in the literature. A recent exception is J. Johannsen and C. Pollett's work [7, where the authors study the $\Delta_{1}^{b}$-bitcomprehension rule in connection with the complexity class $T C^{0}$ of functions computable by uniform threshold circuit families of polynomial size and constant depth. Moreover, both in [2] and in [7] the analysis of those systems has been carried out by means of proof-theoretic methods.

In this paper we shall develop a model-theoretic approach to the investigation of these restricted systems. To this end, the key ingredient is the notion of an $\exists \hat{\Pi}_{i}^{b}$-maximal model, a hierarchical version of the well known notion of an existentially closed model. These models allow us to clarify the relationships between the considered theories and their restricted versions in a particularly simple way. Namely, if $T$ denotes $S_{2}^{i}, T_{2}^{i}$ or $B B \Sigma_{i}^{b}$ and $T^{R}$ (resp. $T^{-}$) denotes its inference rule (resp. parameter free) version, then (see Thm. 11 and Prop. 33)

- every $\exists \hat{\Pi}_{i}^{b}$-maximal model of $T^{R}$ is a model of $T$, and

- every theory extending $T^{-}$is closed under the corresponding inference rule and, so, every $\exists \hat{\Pi}_{i}^{b}$-maximal model of $T^{-}$is a model of $T$.

From these facts we shall derive our main results (see Theorems 3 and 4): (1) $S_{2}^{i}, T_{2}^{i}$ and $B B \Sigma_{i}^{b}$ are $\forall \Sigma_{i}^{b}$-conservative over their inference rule versions; and (2) $S_{2}^{i}, T_{2}^{i}$ are $\exists \forall \Sigma_{i}^{b}$-conservative over their parameter free versions. As far as we know, these results are new, and the $\exists \forall \Sigma_{i}^{b}$-conservation results for parameter free systems improve previous $\forall \Sigma_{i}^{b}$-conservation obtained in [2].

Finally, in Sect. 4 we apply the results obtained for $\Sigma_{1}^{b}$-replacement to the analysis of the $\Delta_{1}^{b}$-bit-comprehension rule $\Delta_{1}^{b}$-BCR. This rule was introduced in [7] to capture the complexity class $T C^{0}$ and is the final refinement of a series of theories introduced in [567] in the quest for natural theories for $T C^{0}$. In 77 it is proved that $T C^{0}$ coincides with the $\Sigma_{1}^{b}$-definable functions of the system $\Delta_{1}^{b}$-CR given by the closure under $\Delta_{1}^{b}-\mathrm{BCR}$ of a certain base theory; and that the $\Sigma_{0}^{b}$ replacement scheme $B B \Sigma_{0}^{b}$ is $\forall \Sigma_{1}^{b}$-conservative over $\Delta_{1}^{b}-\mathrm{CR}$. Here, we prove that $T C^{0}$ also coincides with the $\hat{\Sigma}_{1}^{b}$-definable functions of the (apparently) weaker system $\hat{\Delta}_{1}^{b}$-CR and reformulate this system in terms of $\Sigma_{i}^{b}$-replacement rule, obtaining as a corollary a new proof of the conservation result in 7]. Our analysis is of independent interest in view of the open problems on $\Delta_{1}^{b}$-BCR posed in [7]; however, it also supports Johannsen-Pollett's claim on $\Delta_{1}^{b}$-CR as a minimal natural theory for $T C^{0}$ and makes more transparent the close relationship between $\Delta_{1}^{b}$-bit-comprehension and $\Sigma_{1}^{b}$-replacement.

\section{Fragments of Bounded Arithmetic}

In what follows we state some definitions and results on Bounded Arithmetic that will be used through this paper (see 38 for more information). The first order language of Bounded Arithmetic $\mathcal{L}_{2}$ comprises the usual language of Peano 
Arithmetic $\{0, S,+, \cdot, \leq\}$ together with five new function symbols: $\left\lfloor\frac{x}{2}\right\rfloor,|x|, \#$, $M S P$ and $\bullet$; where $\left\lfloor\frac{x}{2}\right\rfloor$ is $x$ divided by 2 rounded down, $|x|$ is the length of $x$ in binary notation, $x \# y$ is $2^{|x| \cdot|y|}, M S P(x, i)$ is $\left\lfloor\frac{x}{2^{i}}\right\rfloor$, and $x \bullet y$ is the subtraction function. As usual, we also write $x+1$ and $2^{|x|}$ for $S x$ and $1 \# x$, respectively. Bounded formulas of $\mathcal{L}_{2}$ are classified in a hierarchy of sets $\Sigma_{i}^{b}$ and $\Pi_{i}^{b}$ by counting the alternations of bounded quantifiers $(\exists x \leq t, \forall x \leq t)$, ignoring sharply bounded quantifiers $(\exists x \leq|t|, \forall x \leq|t|)$.

The induction axiom for $\varphi(x), I_{\varphi}$, is the formula

$$
\varphi(0) \wedge \forall x(\varphi(x) \rightarrow \varphi(S x)) \rightarrow \forall x \varphi(x)
$$

The length induction axiom for $\varphi(x), L I N D_{\varphi}$, and the double length induction axiom for $\varphi(x), L L I N D_{\varphi}$, are obtained replacing the consequent of $I_{\varphi}$ by $\forall x \varphi(|x|)$ and $\forall x \varphi(\| x||)$, respectively. The polynomial induction axiom for $\varphi(x)$, $P I N D_{\varphi}$, is the formula

$$
\varphi(0) \wedge \forall x\left(\varphi\left(\left\lfloor\frac{x}{2}\right\rfloor\right) \rightarrow \varphi(x)\right) \rightarrow \forall x \varphi(x)
$$

In all cases, $\varphi(x)$ may contain other free variables, which are called parameters. On a par with these induction axioms, we consider induction inference rules. The induction rule for $\varphi(x)$, IR, is

$$
\frac{\varphi(0), \forall x(\varphi(x) \rightarrow \varphi(S x))}{\forall x \varphi(x)}
$$

Similarly, PINDR, LINDR and LLINDR are defined.

$B A S I C$ denotes a finite set of open (quantifier-free) axioms specifying the interpretations of the nonlogical symbols of $\mathcal{L}_{2}$. Following [710, our base theory will be LIOpen $=B A S I C+\left\{L I N D_{\varphi}: \varphi\right.$ is open $\}$. As shown there, LIOpen allows for simple definitions of tuple-encoding and sequence-encoding functions. First, observe that there are $\mathcal{L}_{2}$-terms $\operatorname{Bit}(x, i)$ and $\operatorname{LSP}(x, i)$ returning the value of the bit in the $2^{i}$ position of the binary representation of $x$, and the number consisting of the low $i$ bits of $x$, respectively. The code of a sequence $\left\{b_{0}, b_{1}, \ldots, b_{|s|}\right\}$ with all its elements less than or equal to some $a$ is the number $w<4(a \# 2 s)$ whose binary representation consists of a 1 followed by the binary representations of the elements $b_{i}$ concatenated, each padded with zeroes to length $|a|$ (we shall write $b d(a, s)$ for the bounding term $4(a \# 2 s)$ ). Thus, the $\mathcal{L}_{2}-$ term $\beta_{a}(w, i):=\operatorname{MSP}(L S P(w, S i \cdot|a|), i \cdot|a|)$ returns the $i$-th element of such a sequence. As for tuple-encoding, pairs are coded as $\langle x, y\rangle:=(B+y) \cdot 2 B+(B+x)$, where $B=2^{|\max (x, y)|}$. Then there is an open formula $i s p a i r(u)$ defining the range of the function $\langle x, y\rangle$; and there are terms $(u)_{0},(u)_{1}$ returning the left and right coordinates from a coded pair (see [10] for details). Interestingly, the encoding and decoding functions are all $\mathcal{L}_{2}$-terms so can be used in an $\mathcal{L}_{2}$-formula without altering its quantifier complexity.

The theories we shall deal with are defined as follows. Let $\Gamma$ be a set of formulas and let $\mathbf{E}$ denote one of the schemes: I, PIND, LIND, LLIND. First, 
the theory $\mathbf{E} \Gamma$ is LIOpen $+\left\{\mathbf{E}_{\varphi}: \varphi \in \Gamma\right\}$. Second, the fragment $T+\Gamma-\mathbf{E R}$ is the closure of $T$ under first order logic and nested applications of the $\mathbf{E}$-rule restricted to formulas in $\Gamma$, where $T$ is an arbitrary $\mathcal{L}_{2}$-theory extending LIOpen. Finally, $\mathbf{E} \Gamma^{-}$is LIOpen $+\left\{\mathbf{E}_{\varphi}: \varphi(x) \in \Gamma^{-}\right\}$, where $\varphi(x) \in \Gamma^{-}$means that $x$ is the only free variable occurring in $\varphi$.

With this terminology, the three classic families of Bounded Arithmetic theories $T_{2}^{i}, S_{2}^{i}$ and $R_{2}^{i}$ correspond to $I \Sigma_{i}^{b}, P I N D \Sigma_{i}^{b}$ and $L L I N D \Sigma_{i}^{b}$, respectively. Let us remark, however, that $\mathcal{L}_{2}$ differs from the language of Buss's original theories $S_{2}^{i}$ and $T_{2}^{i}$, which does not include the $M S P$ and $\bullet$ symbols. In addition, Buss's theories are axiomatized over BASIC instead of over LIOpen. But these facts are inessential for sufficiently strong theories since both additional functions are $\Sigma_{1}^{b}$-definable in Buss's $S_{2}^{1}$, and this last theory implies LIOpen.

Bounded formulas of $\mathcal{L}_{2}$ are also classified in a hierarchy of sets strict $\Sigma_{i}^{b}$ (= $\left.\hat{\Sigma}_{i}^{b}\right)$ and strict $\Pi_{i}^{b}\left(=\hat{\Pi}_{i}^{b}\right)$, where no sharply bounded quantifier is allowed to precede a quantifier that is not sharply bounded. Each $\Sigma_{i}^{b}$ (resp. $\Pi_{i}^{b}$ ) formula is equivalent to a $\hat{\Sigma}_{i}^{b}$ (resp. $\hat{\Pi}_{i}^{b}$ ) formula and the $\hat{\Pi}_{i-1}^{b}$-replacement scheme $B B \hat{\Pi}_{i-1}^{b}$ is a natural theory which proves that equivalence. The replacement or bounded collection axiom for a formula $\varphi(x, y)$ and a term $t(x), B B_{\varphi}$, is

$$
\begin{aligned}
\forall x \leq & |s| \exists y \leq t(x) \varphi(x, y) \rightarrow \\
& \exists w<b d\left(t^{*}(|s|), s\right) \forall x \leq|s|\left(\beta_{t^{*}(|s|)}(w, x) \leq t(x) \wedge \varphi\left(x, \beta_{t^{*}(|s|)}\right)\right),
\end{aligned}
$$

where $t^{*}$ denotes an $\mathcal{L}_{2}$-term canonically associated to $t$ so that, provably in LIOpen, $t^{*}$ is monotonic and $t \leq t^{*}$ (see [7]10] for details).

$B B \Gamma$ is LIOpen $+\left\{B B_{\varphi}: \varphi \in \Gamma\right\}$. Similarly, the inference rule versions $T+\Gamma$-BBR are defined. In [10] it is shown that every $\Sigma_{i}^{b}$ formula is provably equivalent in $B B \hat{\Pi}_{i-1}^{b}$ to a $\hat{\Sigma}_{i}^{b}$-formula, and that $P I N D \hat{\Sigma}_{i}^{b}$ implies $B B \hat{\Pi}_{i-1}^{b}$ $(i \geq 1)$. As a consequence, the author obtains the equivalences $T_{2}^{i} \equiv I \hat{\Sigma}_{i}^{b}$ and $S_{2}^{i} \equiv P I N D \hat{\Sigma}_{i}^{b} \equiv L I N D \hat{\Sigma}_{i}^{b}$. Finally, reasoning as in the proof of result 3.2 in [5], it is easy to show that $B B \hat{\Sigma}_{i+1}^{b} \equiv B B \hat{\Pi}_{i}^{b}$, and $T+\hat{\Sigma}_{i+1}^{b}-\mathrm{BBR} \equiv T+\hat{\Pi}_{i}^{b}-\mathrm{BBR}$.

\section{On $\exists \hat{\Pi}_{i}^{b}-$ Maximal Models and Conservation Results}

In this section we present our methods for proving conservation results. To illustrate these methods, we prove that $S_{2}^{i}, T_{2}^{i}$ and $B B \Sigma_{i}^{b}$ are $\forall \Sigma_{i}^{b}$-conservative over their inference rule versions; and we use these results to show that $S_{2}^{i}$ and $T_{2}^{i}$ are $\exists \forall \Sigma_{i}^{b}$-conservative over their parameter free versions. The main idea involves a basic model-theoretic argument: we show that each (countable) model of the weak theory has a $\hat{\Sigma}_{i}^{b}$-elementary extension to a model of the strong theory ( $\mathfrak{B}$ is a $\Gamma$-elementary extension of $\mathfrak{A}, \mathfrak{A} \prec_{\Gamma} \mathfrak{B}$, if $\mathfrak{A} \subseteq \mathfrak{B}$ and, for all $\varphi(\vec{x}) \in \Gamma$ and $\vec{a} \in \mathfrak{A}$, it holds that $\mathfrak{A} \models \varphi(\vec{a}) \Longleftrightarrow \mathfrak{B} \models \varphi(\vec{a}))$. The key ingredient for this construction is the notion of an $\exists \hat{\Pi}_{i}^{b}$-maximal model for a theory $T$.

Definition 1. Let $\mathfrak{A}$ be a model of $T$. We say that $\mathfrak{A}$ is $\exists \hat{\Pi}_{i}^{b}$-maximal for $T$ if, for each $\mathfrak{B}=T$, it holds that $\mathfrak{A} \prec_{\hat{\Sigma}_{i}^{b}} \mathfrak{B} \Longrightarrow \mathfrak{A} \prec_{\exists \hat{\Pi}_{i}^{b}} \mathfrak{B}$. 
This notion is a suitably modified version of the general concept of an existentially closed model. The use of similar notions to prove conservation results for arithmetic systems was presented in a general setting in J. Avigad's 1 (our work is inspired by the methods in that paper). First of all, observe that $\exists \hat{\Pi}_{i}^{b}$ maximal models do exist. The proof is an easy modification of the standard iterative argument to construct existentially closed models.

Proposition 1. Suppose $T$ is $\forall \exists \hat{\Pi}_{i}^{b}$-axiomatizable and $\mathfrak{A}$ is a countable model of $T$. Then there is $\mathfrak{B} \models T$ such that $\mathfrak{A} \prec_{\hat{\Sigma}_{i}^{b}} \mathfrak{B}$ and $\mathfrak{B}$ is $\exists \hat{\Pi}_{i}^{b}$-maximal for $T$.

Next, we prove the main property of these models of interest to us: each $\exists \hat{\Pi}_{i}^{b}$ maximal model for $T+\hat{\Sigma}_{i}^{b}-\mathbf{E R}$ also satisfies the corresponding scheme $\mathbf{E} \hat{\Sigma}_{i}^{b}$. We first need the following result (the proof is a standard compactness argument).

Proposition 2. Let $\mathfrak{A}$ be $\exists \hat{\Pi}_{i}^{b}$-maximal for $T, \vec{a} \in \mathfrak{A}$ and $\varphi(\vec{x}, \vec{v}) \in \hat{\Sigma}_{i}^{b}$ and let $\hat{\Pi}_{i}^{b}-\operatorname{Diag}(\mathfrak{A})$ denote the set of all the $\hat{\Pi}_{i}^{b}$-formulas (with parameters in $\mathfrak{A}$ ) valid in $\mathfrak{A}$. The following conditions are equivalent.

1. $\mathfrak{A} \models \forall \vec{x} \varphi(\vec{x}, \vec{a})$.

2. There is $\theta(\vec{a}, \vec{b})$ in $\hat{\Pi}_{i}^{b}-\operatorname{Diag}(\mathfrak{A})$ satisfying $T+\theta(\vec{a}, \vec{b}) \vdash \forall \vec{x} \varphi(\vec{x}, \vec{a})$.

Theorem 1. Let $\mathbf{E}$ denote one of the following schemes: BB, I, PIND, LIND, $L L I N D$. If $\mathfrak{A}$ is $\exists \hat{\Pi}_{i}^{b}-$ maximal for $T+\hat{\Sigma}_{i}^{b}-\mathbf{E R}$, then $\mathfrak{A} \models \mathbf{E} \hat{\Sigma}_{i}^{b}$.

Proof. (Collection scheme): Assume $\mathfrak{A}$ is $\exists \hat{\Pi}_{i}^{b}$-maximal for $T+\hat{\Sigma}_{i}^{b}-\mathrm{BBR}$ and $\mathfrak{A} \models \forall x \leq|s| \exists y \leq t \varphi(x, y, a)$, where $\varphi(x, y, v) \in \hat{\Sigma}_{i}^{b}, a \in \mathfrak{A}$ and $s, t$ are $\mathcal{L}_{2}-$ terms (for notational simplicity we omit the possible parameters in $t, s$ ). By Proposition 2 there are $b \in \mathfrak{A}$ and $\theta(v, u)$ in $\hat{\Pi}_{i}^{b}$ such that $\mathfrak{A} \models \theta(a, b)$, and $\left(T+\hat{\Sigma}_{i}^{b}-\mathrm{BBR}\right)+\theta(a, b) \vdash \forall x \leq|s| \exists y \leq t \varphi(x, y, a)$. So, $T+\hat{\Sigma}_{i}^{b}-\mathrm{BBR} \vdash \theta(v, u) \rightarrow$ $\forall x \leq|s| \exists y \leq t \varphi(x, y, v)$. Define $\delta(x, y, v, u)$ to be $\neg \theta(v, u) \vee \varphi(x, y, v)$. Clearly, $\delta$ is $\hat{\Sigma}_{i}^{b}$ and $T+\hat{\Sigma}_{i}^{b}$-BBR proves the antecedent of the bounded collection axiom for $\delta(x, y)$. Applying $\hat{\Sigma}_{i}^{b}-\mathrm{BBR}$ in $\mathfrak{A}$ and taking $v=a$ and $u=b$, we get

$$
\mathfrak{A} \models \exists w<b d\left(t^{*}(|s|), s\right) \forall x \leq|s|\left(\beta_{t^{*}(|s|)}(w, x) \leq t \wedge \delta\left(x, \beta_{t^{*}(|s|)}(w, x), a, b\right)\right)
$$

Since $\mathfrak{A} \models \theta(a, b), \mathfrak{A} \models \delta(x, y, a, b) \rightarrow \varphi(x, y, a)$ and hence the consequent of the bounded collection axiom for $\delta(x, y, a)$ is true in $\mathfrak{A}$.

(Induction schemes): We only write the proof for the usual induction scheme $I$, the remaining cases being analogous. Assume $\mathfrak{A}$ is $\exists \hat{\Pi}_{i}^{b}$-maximal for $T+\hat{\Sigma}_{i}^{b}$-IR. To prove that $\mathfrak{A} \models I \hat{\Sigma}_{i}^{b}$, assume $\mathfrak{A} \models \varphi(0, a) \wedge \forall x(\varphi(x, a) \rightarrow \varphi(x+1, a))$, where $\varphi(x, v) \in \hat{\Sigma}_{i}^{b}$ and $a \in \mathfrak{A}$. We must show $\mathfrak{A} \models \forall x \varphi(x, a)$. Put $\varphi(x, v)$ as $\exists y \leq t(x, v) \varphi_{0}(x, y, v)$, where $\varphi_{0}(x, y, v) \in \hat{\Pi}_{i-1}^{b}$ and $t(x, v)$ is a term. By prenex operations, the antecedent of the induction axiom for $\varphi$ can be reexpressed as

$$
\forall x \forall y\left[\varphi(0, a) \wedge\left(\neg(y \leq t(x, a)) \vee \neg \varphi_{0}(x, y, a) \vee \varphi(x+1, a)\right)\right]
$$

Let us denote by $\psi(x, y, a)$ the $\hat{\Sigma}_{i}^{b}$-formula in brackets [] above. Since $\mathfrak{A}$ is $\exists \hat{\Pi}_{i}^{b}$ maximal for $T+\hat{\Sigma}_{i}^{b}$-IR and $\mathfrak{A} \models \forall x, y \psi(x, y, a)$, by Proposition 2 it follows that 
there are $b \in \mathfrak{A}$ and $\theta(v, u) \in \hat{\Pi}_{i}^{b}$ satisfying $\mathfrak{A} \models \theta(a, b)$, and $\left(T+\hat{\Sigma}_{i}^{b}-\mathrm{IR}\right)+$ $\theta(a, b) \vdash \forall x, y \psi(x, y, a)$. Hence,

$$
T+\hat{\Sigma}_{i}^{b}-\mathrm{IR} \vdash \theta(v, u) \rightarrow(\varphi(0, v) \wedge \forall x(\varphi(x, v) \rightarrow \varphi(x+1, v)))
$$

Now define $\delta(x, v, u)$ to be the $\hat{\Sigma}_{i}^{b}$-formula $\neg \theta(v, u) \vee \varphi(x, v)$. Clearly, $T+\hat{\Sigma}_{i}^{b}-\mathrm{IR}$ proves the antecedent of the induction axiom for $\delta(x, v, u)$. By applying $\hat{\Sigma}_{i}^{b}-\mathrm{IR}$, we get $\mathfrak{A} \models \forall x, v, u \delta(x, v, u)$. In particular, $\mathfrak{A} \models \forall x(\neg \theta(a, b) \vee \varphi(x, a))$, and hence $\mathfrak{A} \models \forall x \varphi(x, a)$ since $\theta(a, b)$ is true in $\mathfrak{A}$.

Combining Proposition 1 and Theorem 1, we can derive our $\forall \Sigma_{i}^{b}$-conservation results. The proof is in two steps. First, we prove this conservation result only for $\forall \hat{\Sigma}_{i}^{b}$-formulas. Second, we show how to extend it to general $\forall \Sigma_{i}^{b}$-formulas.

Theorem 2. Let $\mathbf{E}$ denote one of the following schemes: BB, I, PIND, LIND, $L L I N D$ and let $T$ be a $\forall \exists \hat{\Pi}_{i}^{b}$-axiomatizable theory. Then $T+\mathbf{E} \hat{\Sigma}_{i}^{b}$ is $\forall \hat{\Sigma}_{i}^{b}$ conservative over $T+\hat{\Sigma}_{i}^{b}-\mathbf{E R}$.

Proof. By contradiction, assume $T+\mathbf{E} \hat{\Sigma}_{i}^{b} \vdash \varphi$ but $T+\hat{\Sigma}_{i}^{b}-\mathbf{E R} \forall \varphi$, where $\varphi \in \forall \hat{\Sigma}_{i}^{b}$. Let $\mathfrak{A}$ be a countable model of $\left(T+\hat{\Sigma}_{i}^{b}-\mathbf{E R}\right)+\neg \varphi$. Since $T$ is $\forall \exists \hat{\Pi}_{i}^{b}$-axiomatizable, so is $T+\hat{\Sigma}_{i}^{b}-\mathbf{E R}$ (for $\mathbf{E}=B B$, recall that $\hat{\Sigma}_{i}^{b}-\mathrm{BBR}$ and $\hat{\Pi}_{i-1}^{b}-\mathrm{BBR}$ are equivalent rules). By Proposition 1 there is $\mathfrak{B} \models T+\hat{\Sigma}_{i}^{b}-\mathbf{E R}$ such that $\mathfrak{A} \prec_{\hat{\Sigma}_{i}^{b}} \mathfrak{B}$ and $\mathfrak{B}$ is $\exists \hat{\Pi}_{i}^{b}$-maximal for $T+\hat{\Sigma}_{i}^{b}-\mathbf{E R}$. From Theorem 1 it follows that $\mathfrak{B} \models \mathbf{E} \hat{\Sigma}_{i}^{b}$. Hence, $\mathfrak{B} \models T+\mathbf{E} \hat{\Sigma}_{i}^{b}+\neg \varphi$, which is a contradiction.

Since $T_{2}^{i}$ and $S_{2}^{i}$ are $\forall \hat{\Sigma}_{i+1}^{b}$-axiomatizable, a first application of Theorem 2 is the following strengthening of the well known facts that $S_{2}^{i+1}$ implies $T_{2}^{i}$ and $R_{2}^{i+1}$ implies $S_{2}^{i}$, and of theorem 68 in [10] stating that $B B \hat{\Sigma}_{i+1}^{b}$ implies $S_{2}^{i}$.

\section{Corollary 1}

1. LIOpen $+\hat{\Sigma}_{i+1}^{b}-$ LINDR implies $T_{2}^{i}$.

2. Both LIOpen $+\hat{\Sigma}_{i+1}^{b}$ LLINDR and LIOpen $+\hat{\Sigma}_{i+1}^{b}-$ BBR imply $S_{2}^{i}$.

To extend previous conservation result to $\forall \Sigma_{i}^{b}$-formulas, we need the following lemma (the proof is by induction on the complexity of $\Sigma_{i}^{b}$-formulas).

Lemma 1. $(i \geq 1)$ Let $\varphi(\vec{v}) \in \Sigma_{i}^{b}$. There exists $\hat{\varphi}(\vec{v}) \in \hat{\Sigma}_{i}^{b}$ such that:
(1) $B B \hat{\Pi}_{i-1}^{b} \vdash \varphi(\vec{v}) \leftrightarrow \hat{\varphi}(\vec{v})$, and
(2) $B B \hat{\Pi}_{i-2}^{b} \vdash \hat{\varphi}(\vec{v}) \rightarrow \varphi(\vec{v})$.

(For $i=1, B B \hat{\Pi}_{-1}^{b}$ denotes LIOpen.)

Theorem 3. Let $\mathbf{E}$ denote one of the following schemes: BB, I, PIND, LIND. Then, LIOpen $+\hat{\Sigma}_{i}^{b}-\mathbf{E R}$ axiomatizes the $\forall \Sigma_{i}^{b}-$ consequences of $\mathbf{E} \Sigma_{i}^{b}$.

Proof. Assume $\mathbf{E} \Sigma_{i}^{b} \vdash \forall \vec{v} \varphi(\vec{v})$, where $\varphi \in \Sigma_{i}^{b}$. Let $\hat{\varphi}(\vec{v}) \in \hat{\Sigma}_{i}^{b}$ as in Lemma 1 . Since $S_{2}^{i}$ implies $B B \Pi_{i-1}^{b}$ (see [3]), E $\Sigma_{i}^{b}$ implies $B B \Pi_{i-1}^{b}$ and LIOpen $+\hat{\Sigma}_{i}^{b}-\mathbf{E R}$ implies $B B \Pi_{i-2}^{b}$ by Corollary 1. Hence, $\mathbf{E} \Sigma_{i}^{b} \vdash \forall \vec{v} \hat{\varphi}(\vec{v})$ and LIOpen $+\hat{\Sigma}_{i}^{b}-\mathbf{E R} \vdash$ $\hat{\varphi}(\vec{v}) \rightarrow \varphi(\vec{v})$. So, this last theory proves $\forall \vec{v} \varphi(\vec{v})$ by Theorem 2 . 
In what follows we deal with parameter free versions of $T_{2}^{i}$ and $S_{2}^{i}$. Notice that there are two natural candidates for their parameter free counterparts: restricting the axiom scheme to parameter free $\Sigma_{i}^{b}$-formulas, or to strict parameter free $\Sigma_{i}^{b}$ formulas. Since we are interested in conservation results over these theories, we choose the weakest ones to make the results stronger. That is, we fix $T_{2}^{i,-} \equiv$ $I \hat{\Sigma}_{i}^{b,-}$ and $S_{2}^{i,-} \equiv P I N D \hat{\Sigma}_{i}^{b,-}$. We derive the conservation theorems from our previous work on inference rules. The key observation is the following:

\section{Proposition 3}

1. If $T$ implies $T_{2}^{i,-}$ then $T$ is closed under $\hat{\Sigma}_{i}^{b}-\mathrm{IR}$.

2. $(i \geq 1)$ If $T$ implies PIND $\Sigma_{1}^{b,-}+S_{2}^{i,-}$ then $T$ is closed under $\hat{\Sigma}_{i}^{b}$-PINDR.

Proof. (1): Assume $T$ proves $\varphi(0, v) \wedge \forall x(\varphi(x, v) \rightarrow \varphi(x+1, v))$, where $\varphi(x, v)$ is $\hat{\Sigma}_{i}^{b}$. We must show $T \vdash \forall v \forall x \varphi(x, v)$. The idea is to codify the parameter $v$ and the induction variable $x$ in a single variable $u$ using the pairing function and to apply $I \hat{\Sigma}_{i}^{b,-}$. To this end, define $\theta(u)$ to be the following $\hat{\Sigma}_{i}^{b}$-formula:

$$
\left(i \operatorname{spair}(u) \wedge(u)_{0}<(u)_{1} \wedge i \operatorname{spair}\left((u)_{1}\right)\right) \rightarrow \varphi\left((u)_{0},(u)_{1,1}\right)
$$

Trivially, $T \vdash \theta(0)$ since $\neg$ ispair $(0)$. Let us see that $T \vdash \forall u(\theta(u) \rightarrow \theta(u+1))$. Reasoning in $T$, we assume $\theta(u)$ and $\left(i \operatorname{spair}\left(u^{\prime}\right) \wedge\left(u^{\prime}\right)_{0}<\left(u^{\prime}\right)_{1} \wedge i \operatorname{spair}\left(\left(u^{\prime}\right)_{1}\right)\right.$, where $u^{\prime}=u+1$. We must show $\varphi\left(\left(u^{\prime}\right)_{0},\left(u^{\prime}\right)_{1,1}\right)$.

Case 1: $\left(u^{\prime}\right)_{0}=0$. Then $\varphi\left(0,\left(u^{\prime}\right)_{1,1}\right)$ since $T \vdash \forall v \varphi(0, v)$.

Case 2: $\left(u^{\prime}\right)_{0}>0$. Since $\left(u^{\prime}\right)_{0}<\left(u^{\prime}\right)_{1}, \max \left(\left(u^{\prime}\right)_{0}-1,\left(u^{\prime}\right)_{1}\right)=\left(u^{\prime}\right)_{1}$ and hence by the definition of the pairing function $u$ codifies the pair $\left\langle\left(u^{\prime}\right)_{0}-1,\left(u^{\prime}\right)_{1}\right\rangle$ (that is, $(u)_{0}=\left(u^{\prime}\right)_{0}-1$ and $\left.(u)_{1}=\left(u^{\prime}\right)_{1}\right)$. Consequently, from $\theta(u)$ it follows $\varphi\left(\left(u^{\prime}\right)_{0}-1,\left(u^{\prime}\right)_{1,1}\right)$ and hence $\varphi\left(\left(u^{\prime}\right)_{0},\left(u^{\prime}\right)_{1,1}\right)$ since $T \vdash \varphi(x, v) \rightarrow \varphi(x+1, v)$.

From the induction axiom for $\theta(u)$ (available in $T$ since it contains $I \hat{\Sigma}_{i}^{b,-}$ ) it follows that $T \vdash \forall u \theta(u)$. To show $T \vdash \forall v \forall x \varphi(x, v)$, observe that $\varphi(x, v)$ can be inferred from $\theta(\langle x,\langle x, v\rangle\rangle)$.

(2): The proof is similar to that of 1 but now we need to define a new tupleencoding function compatible with the PIND axioms: roughly speaking, if $u$ codifies the pair $(x, \vec{v})$ and $x>0$, then $\left\lfloor\frac{u}{2}\right\rfloor$ must codify the pair $\left(\left\lfloor\frac{x}{2}\right\rfloor, \vec{v}\right)$. In $[2]$ Bloch proposed the following encoding function satisfying that property:

$$
[x, v, z]=u \equiv\left\{\begin{array}{l}
|v|<z^{2} \leq|u|<(z+1)^{2} \wedge \\
u=\operatorname{Concat}\left(v+2^{\min \left(z^{2},|u|\right)}, x+2^{|x|}\right)
\end{array}\right.
$$

where $\operatorname{Concat}(x, y)=x \cdot 2^{|y| \bullet^{-1}}+y \bullet 2^{|y| \bullet^{-1}}$. In words, we pad $v$ to length $z^{2}$ and concatenate the result with $x$ (notice that the Concat function operates on bit-strings rather than on binary numbers, that is, Concat $(1 x, 1 y)=1 x y)$. Observe that the encoding function $[x, v, z]$ itself is not total, but it is total for all $z$ sufficiently large. Namely, as shown in [2], PIND $\Sigma_{1}^{b,-}$ proves:

(a) $|x| \leq 2 z \wedge|v|<z^{2} \rightarrow \exists ! u([x, v, z]=u)$,

(b) $u>0 \rightarrow \exists ! x, v, z \leq u([x, v, z]=u)$, and

(c) $u=[x, v, z] \wedge x>0 \rightarrow\left\lfloor\frac{u}{2}\right\rfloor=\left[\left\lfloor\frac{x}{2}\right\rfloor, v, z\right]$ 
Equipped with this encoding function, we can infer the PIND axiom for the $\hat{\Sigma}_{i}^{b}$-formula $\varphi(x, v)$ from the PIND axiom for the (parameter free) $\hat{\Sigma}_{i}^{b}$-formula $\theta(u) \equiv u>0 \rightarrow \exists x, v, z \leq u([x, v, z]=u \wedge \varphi(x, v))$.

Observe that from the previous result and Theorem 2 it immediately follows that $T_{2}^{i+1,-}$ implies $T_{2}^{i}$ and that PIND $\Sigma_{1}^{b,-}+S_{2}^{i+1,-}$ implies $S_{2}^{i}$.

\section{Theorem 4}

1. $T_{2}^{i}$ is $\exists \forall \Sigma_{i}^{b}$-conservative over $T_{2}^{i,-}$.

2. $(i \geq 1) S_{2}^{i}$ is $\exists \forall \Sigma_{i}^{b}$-conservative over PIND $\Sigma_{1}^{b,-}+S_{2}^{i,-}$.

Proof. Using Lemma 1 as in Theorem 3, it suffices to show $\exists \forall \hat{\Sigma}_{i}^{b}$-conservation. We only write the proof of 1 . Assume $\varphi$ is an $\exists \forall \hat{\Sigma}_{i}^{b}$-sentence such that $T_{2}^{i} \vdash \varphi$ but $T_{2}^{i,-} \forall \varphi$. Then $T=T_{2}^{i,-}+\neg \varphi$ is consistent and $\forall \exists \hat{\Pi}_{i}^{b}$-axiomatizable. Let $\mathfrak{A}$ be an $\exists \hat{\Pi}_{i}^{b}$-maximal model for $T$. By Proposition 3 , $T$ is closed under $\hat{\Sigma}_{i}^{b}-$ IR. Hence, $\mathfrak{A} \models T+T_{2}^{i}$ by Theorem 11. So, $\mathfrak{A} \models T_{2}^{i}+\neg \varphi$, which is a contradiction.

As for parameter free $B B \Sigma_{i}^{b}$, we can prove that $B B \Sigma_{i}^{b}$ is $\exists \forall \Sigma_{i}^{b}$-conservative over $U B B \hat{\Sigma}_{i}^{b}$ as in Theorem $4\left(U B B_{\varphi}\right.$ is obtained quantifying universally the parameters of $\varphi(x, y)$ in both the antecedent and the consequent of $\left.B B_{\varphi}\right)$.

\section{On Replacement and Bit-Comprehension Rules}

In this section we shall study an inference rule closely tied to $\Sigma_{1}^{b}$-replacement: $\Delta_{1}^{b}$-bit-comprehension rule. This rule was defined in [7] as follows:

$$
\Delta_{1}^{b}-\mathrm{BCR}: \quad \frac{\varphi(x) \leftrightarrow \psi(x)}{\exists y<2^{|u|} \forall x<|u|(\operatorname{Bit}(y, x)=1 \leftrightarrow \varphi(x))}
$$

where $\varphi(x) \in \Sigma_{1}^{b}$ and $\psi(x) \in \Pi_{1}^{b}$. In [7], it is proved that $B B \Sigma_{0}^{b}$ (denoted there by $C_{2}^{0}$ ) is a $\forall \Sigma_{1}^{b}$-conservative extension of $\Delta_{1}^{b}$-CR (the theory LIOpen $+\Delta_{1}^{b}-\mathrm{BCR}$ ). So, in view of Theorem 3 , it is natural to investigate the relationship between $\Delta_{1}^{b}-\mathrm{BCR}$ and $\hat{\Sigma}_{1}^{b}$-BBR. In this section, we consider the apparently weaker rule for strict formulas $\hat{\Delta}_{1}^{b}$-BCR and show that LIOpen $+\hat{\Delta}_{1}^{b}$-BCR (denoted in what follows by $\hat{\Delta}_{1}^{b}-\mathrm{CR}$ ) is equivalent to LIOpen $+\hat{\Sigma}_{1}^{b}-\mathrm{BBR}$, see Theorem 5 . In fact, over LIOpen, the four rules $\Sigma_{1}^{b}-\mathrm{BBR}, \hat{\Sigma}_{1}^{b}-\mathrm{BBR}, \Delta_{1}^{b}-\mathrm{BCR}$ and $\hat{\Delta}_{1}^{b}$-BCR are equivalent and, by Theorem 3 provide axiomatizations of the $\forall \Sigma_{1}^{b}$-consequences of $B B \Sigma_{1}^{b}$. Moreover, in [9], answering a question posed in [7, it is shown that $\Delta_{1}^{b}$-CR is finitely axiomatizable; so, a finite number of nested applications of any of the rules above axiomatizes the $\forall \Sigma_{1}^{b}$-consequences of $B B \Sigma_{1}^{b}$. However,

Problem 1. Is LIOpen $+\hat{\Sigma}_{1}^{b}-B B R$ equivalent to [LIOpen; $\left.\hat{\Sigma}_{1}^{b}-B B R\right]$, the closure of LIOpen under first order logic and unnested applications of $\hat{\Sigma}_{1}^{b}-B B R$ ?

Our work suggests a positive answer to Problem 1 since this is the case for the analogous problem for collection rule in the usual language of Peano Arithmetic.

Now we prove that LIOpen $+\hat{\Sigma}_{1}^{b}-\mathrm{BBR}$ and $\hat{\Delta}_{1}^{b}-\mathrm{CR}$ are equivalent. Our work also provides a new proof of Johannsen-Pollett's conservation theorem. 
Firstly, observe that it can be easily shown that LIOpen $+\hat{\Sigma}_{1}^{b}-\mathrm{BBR}$ is closed under $\hat{\Delta}_{1}^{b}$-BCR. On the other hand, since $C_{2}^{0}$ coincides with $B B \Sigma_{0}^{b}$, by Theorem 2. $C_{2}^{0}$ is $\forall \hat{\Sigma}_{1}^{b}$ conservative over LIOpen $+\hat{\Sigma}_{1}^{b}-\mathrm{BBR}$. So, in order to simultaneously get the equivalence of LIOpen $+\hat{\Sigma}_{1}^{b}-\mathrm{BBR}$ and $\hat{\Delta}_{1}^{b}-\mathrm{CR}$, and Johannsen-Pollett's theorem, it suffices to prove that $\hat{\Delta}_{1}^{b}-\mathrm{CR}$ is closed under $\hat{\Sigma}_{1}^{b}$-BBR. Next two lemmas are the key ingredients of the proof. The first one provides a weak form of replacement available in $\hat{\Delta}_{1}^{b}-\mathrm{CR}$ (the proof is straightforward and we omit it). The second one is a selection (or witnessing) principle for $\hat{\Delta}_{1}^{b}-\mathrm{CR}$.

Lemma 2. Let $\varphi(x, y) \in \hat{\Sigma}_{1}^{b}$ such that $\hat{\Delta}_{1}^{b}-C R \vdash \forall x \leq|s| \exists ! y \leq t \varphi(x, y)$. Then

$$
\hat{\Delta}_{1}^{b}-C R \vdash \exists w<b d\left(t^{*}(|s|), s\right) \forall x \leq|s|\left(\beta_{t^{*}(|s|)}(w, x) \leq t \wedge \varphi\left(x, \beta_{t^{*}(|s|)}(w, x)\right)\right)
$$

Lemma 3. Let $\varphi(x, y) \in \hat{\Sigma}_{1}^{b}$ such that $\hat{\Delta}_{1}^{b}-C R \vdash \forall x \exists y \leq t \varphi(x, y)$, then there exists $\psi(x, y) \in \hat{\Sigma}_{1}^{b}$ such that $\hat{\Delta}_{1}^{b}-C R$ proves

$$
\text { (1) } \forall x \exists ! y \leq t \psi(x, y), \quad \text { and } \quad(2) \quad \forall x \forall y(\psi(x, y) \rightarrow \varphi(x, y))
$$

Proof. (Sketch) The proof we present here leans upon an analysis of the class of $\hat{\Sigma}_{1}^{b}$-definable functions $\hat{\Delta}_{1}^{b}-\mathrm{CR}$. We refine corollary 1 in 7 and prove that $T C^{0}$ is the class of $\hat{\Sigma}_{1}^{b}$-definable functions of $\hat{\Delta}_{1}^{b}-\mathrm{CR}$. The basic result is a machineindependent characterization of $T C^{0}$ given by Clote and Takeuti in [4:

Let $B F$ be the set of basic functions $\left\{o, s_{0}, s_{1}, \#, \times,|\cdot|\right\} \cup\left\{\Pi_{i}^{n}: 1 \leq i \leq n\right\}$, where $o(x)=0, s_{0}(s)=2 x, s_{1}(x)=2 x+1,|x|=\left\lceil\log _{2}(x+1)\right\rceil, x \# y=2^{|x| \cdot|y|}$, $\times$ denotes the usual product and $\Pi_{i}^{n}\left(x_{1}, \ldots, x_{n}\right)=x_{i}$.

Given $g: \omega^{n} \rightarrow \omega$ and $h_{0}, h_{1}: \omega^{n+1} \rightarrow\{0,1\}$, a function $f$ is defined by concatenation recursion on notation (CRN) from $g, h_{0}$ and $h_{1}$ if

$$
\begin{aligned}
f(0, \vec{x}) & =g(\vec{x}) \\
f(2 n, \vec{x}) & =2 \cdot f(n, \vec{x})+h_{0}(n, \vec{x}), \quad \text { provided } n \neq 0 \\
f(2 n+1, \vec{x}) & =2 \cdot f(n, \vec{x})+h_{1}(n, \vec{x})
\end{aligned}
$$

Clote and Takeuti proved that $T C^{0}$ is the smallest class of functions containing $B F$ and closed under composition and CRN. In order to show that every function in $T C^{0}$ is $\hat{\Sigma}_{1}^{b}$-definable in $\hat{\Delta}_{1}^{b}$-CR, we show a stronger technical result:

For each function $f \in T C^{0}$ there exist a formula $\psi\left(\vec{x}, y, z_{1}, \ldots, z_{n}\right) \in \Sigma_{0}^{b}$ and terms $t(x), t_{1}(x, y), t_{2}\left(x, y, z_{1}\right), \ldots, t_{n}\left(x, y, z_{1}, \ldots, z_{n-1}\right)$ such that the $\hat{\Sigma}_{1}^{b}$ formula $\exists z_{1} \leq t_{1} \ldots \exists z_{n} \leq t_{n} \psi(x, y, \vec{z})$ defines $f$ in the standard model and

$$
\text { LIOpen }+\hat{\Delta}_{1}^{b}-\mathrm{BCR} \vdash \forall \vec{x} \exists ! y \leq t \exists ! z_{1} \leq t_{1} \ldots \exists ! z_{n} \leq t_{n} \psi(x, y, \vec{z})
$$

The proof proceeds by induction, using Clote-Takeuti's characterization of $T C^{0}$. The claim obviously holds for the basic functions and for $f$ defined by composition from functions verifying the claim. So it suffices to prove the result for functions defined by CRN and this can be done as in theorem 4 in 7].

It is not difficult to verify that, if $f \in T C^{0}$ is defined by CRN from $g, h_{0}$ and $h_{1}$ then the proof of the previous technical result provide $\hat{\Sigma}_{1}^{b}$-formulas defining the functions involved and such that the relations stated by the recursion equations of $\mathrm{CRN}$ can be proved in $\hat{\Delta}_{1}^{b}-\mathrm{CR}$. Bearing this fact in mind, we can introduce a universally axiomatized and conservative extension of $\hat{\Delta}_{1}^{b}-\mathrm{CR}$, denoted by 
CRNA. This universal theory can be defined in such a way that the functions in $T C^{0}$ are defined by terms of CRNA. In this way we can prove that every function $\hat{\Sigma}_{1}^{b}$-definable in $\hat{\Delta}_{1}^{b}$-CR is in $T C^{0}$ by a typical application of Herbrand's theorem. The whole argument is very similar to the Herbrand analyses of $S_{2}^{i}$ developed by W. Sieg in 11.

Finally, we derive Lemma 3 from Herbrand's theorem applied to CRNA.

Theorem 5. The theories $\hat{\Delta}_{1}^{b}-C R$ and LIOpen $+\Sigma_{1}^{b}-B B R$ are equivalent and axiomatize the class of the $\forall \Sigma_{1}^{b}$-consequences of $C_{2}^{0}$.

Proof. Observe that $C_{2}^{0}$ extends LIOpen $+\Sigma_{1}^{b}$-BBR, which in turn extends $\hat{\Delta}_{1}^{b}$-CR; so, since LIOpen $+\Sigma_{1}^{b}$-BBR is $\forall \Sigma_{1}^{b}$-axiomatized, it suffices to prove that $C_{2}^{0}$ is $\forall \Sigma_{1}^{b}$-conservative over $\hat{\Delta}_{1}^{b}$-CR. Finally, by Theorem 3 it is enough to show that $\hat{\Delta}_{1}^{b}-\mathrm{CR}$ is closed under $\hat{\Sigma}_{1}^{b}$-BBR. Let us work in $\hat{\Delta}_{1}^{b}-\mathrm{CR}$.

Let $\varphi(x, y) \in \hat{\Sigma}_{1}^{b}$ and $t, s$ be terms such that $\forall x \leq|s| \exists y \leq t \varphi(x, y)$. Define $\theta(x, y) \in \hat{\Sigma}_{1}^{b}$ to be $(x>|s| \wedge y=0) \vee(x \leq|s| \wedge \varphi(x, y))$. Then $\forall x \exists y \leq t \theta(x, y)$ and, by Lemma 3, there is $\psi(x, y) \in \hat{\Sigma}_{1}^{b}$ such that (1) $\forall x \exists ! y \leq t \psi(x, y)$, and (2) $\forall x \forall y(\psi(x, y) \rightarrow \theta(x, y))$. By (1) and Lemma 2, it holds that

$$
\exists w<b d\left(t^{*}(|s|), s\right) \forall x \leq|s|\left(\beta_{t^{*}(|s|)}(w, x) \leq t \wedge \psi\left(x, \beta_{t^{*}(|s|)}(w, x)\right)\right)
$$

Hence, $\exists w<b d\left(t^{*}(|s|), s\right) \forall x \leq|s|\left(\beta_{t^{*}(|s|)}(w, x) \leq t \wedge \varphi\left(x, \beta_{t^{*}(|s|)}(w, x)\right)\right)$, since, by (2) and the definition of $\theta$, we have $x \leq|s| \wedge \psi(x, y) \rightarrow \varphi(x, y)$.

Corollary 2. (Johannsen-Pollett) $C_{2}^{0}$ is $\forall \Sigma_{1}^{b}$-conservative over $\Delta_{1}^{b}-C R$.

\section{References}

1. Avigad, J.: Saturated models of universal theories. Annals of Pure and Applied Logic 118, 219-234 (2002)

2. Bloch, S.: Divide and Conquer in Parallel Complexity and Proof Theory, Ph. D. Thesis. University of California, San Diego (1992)

3. Buss, S.: Bounded Arithmetic. Bibliopolis, Napoli (1986)

4. Clote, P., Takeuti, G.: First order bounded artihmetic and small boolean circuit complexity classes. In: Clote, P., Remmel, J (eds.) Feasible Mathematics II, pp. 154-218. Birkhäuser, Boston (1995)

5. Johannsen, J.: A Bounded Arithmetic Theory for Constant Depth Threshold Circuits. In: Gödel'96. Lecture Notes in Logic, vol. 6, pp. 224-234. Springer, Heidelberg (1996)

6. Johannsen, J., Pollett, C.: On Proofs About Threshold Circuits and Counting Hierarchies. In: Proc. 13th IEEE Symposium on Logic in Computer Science (1998)

7. Johannsen, J., Pollett, C.: On the $\Delta_{1}^{b}$-Bit-Comprehension Rule. Logic Colloquium'98. Lecture Notes in Logic, ASL 13, 262-279 (2000)

8. Krajíček, J.: Bounded Arithmetic, Propositional Logic, and Complexity Theory. Cambridge University Press, Cambridge (1995)

9. Nguyen, P., Cook, S.: Theories for $T C^{0}$ and other small complexity classes. Logical Methods in Computer Science 2, 1-40 (2006)

10. Pollett, C.: Structure and Definability in General Bounded Arithmetic Theories. Annals of Pure and Applied Logic 100, 189-245 (1999)

11. Sieg, W.: Herbrand Analyses. Archive for Mathematical Logic 30, 409-441 (1991) 